\title{
Ischemic Change
}

National Cancer Institute

\section{Source}

National Cancer Institute. Ischemic Change. NCI Thesaurus. Code C82934.

Cellular damage due to diminished oxygen supply to the tissues. The changes may be mild and associated with chronic inflammation, or severe and associated with ulceration, necrosis, and hemorrhage. 